\title{
THE RADIO AND OPTICAL PROPERTIES OF QUASARS
}

\author{
C.D. IMPEY AND E.J. HOOPER \\ Steward Observatory \\ University of Arizona, Tucson, AZ 85721, USA \\ C.B. FOLTZ \\ Multiple Mirror Telescope Observatory \\ University of Arizona, Tucson, AZ 85721, USA \\ AND \\ P.C. HEWETT \\ Institute of Astronomy \\ Madingley Road, Cambridge CB3 OHA, England
}

\section{Introduction}

The relationship between the radio and optical emission of quasars was first studied using radio-selected objects, which generally had high radio luminosities due to the relatively low sensitivity limits of the surveys. More recently, radio follow-up observations have been made of optical surveys surveys in other wavebands. Taken together, the two survey methods have detected quasars with a range of over 6 orders of magnitude in radio luminosity. We report here new observations with the Very Large Array (VLA) of 103 quasars from the Large Bright Quasar Survey (LBQS), with absolute magnitudes in the range $-25<M_{\mathrm{B}}<-23$ and redshifts in the range $0.2<z<1.1$. This can be combined with our previous LBQS observations (Paper I, Paper II) to yield 359 quasars, the largest sample of sensitive radio observations of optically-selected quasars in a single survey.

\section{Results}

First, the distribution of radio luminosity does not depend on absolute magnitude over most of the range of $M_{\mathrm{B}}$ in the LBQS. The radio-loud fraction remains constant at $\approx 10 \%$ for $-28<M_{\mathrm{B}}<-23$ but rises to $\approx 30 \%$ at brighter absolute magnitudes. Second, the radio properties of the LBQS 
quasars as a function of $M_{\mathrm{B}}$ are consistent with the existence of two radio emission mechanisms, one correlated with optical luminosity, the other independent. The sudden decrease in radio-loud fraction for $M_{\mathrm{B}}$ fainter than -24 observed in the Palomar-Greeen (PG) sample is not present in the expanded LBQS sample. A selection effect to which the LBQS is mostly immune is the likely cause of the decrease in the PG sample.

The radio-loud fraction in optically selected quasars appears to be unevolving at a value of $\approx 10 \%$, aside from a modest increase at $z \sim 1$, from $z=0.2$ to redshifts approaching 5 , based on the LBQS and three high- $z$ optically selected samples. A model based on a radio luminosity function derived from radio-selected quasars (Dunlop \& Peacock 1990) matches the data well, including the modest rise around $z=1$, at all except the highest redshifts. The high radio-loud fraction in the PG sample remains unexplained (Kellerman et al. 1989). Fourth, the radio properties of the X-ray selected EMSS also differ from those of the LBQS (Stocke et al. 1991). The rapid rise in radio-loud fraction observed for $M_{\mathrm{B}} \approx-24$ is primarily an evolutionary effect resulting from a well-established correlation between $\mathrm{X}$-ray and radio luminosity. The rise in radio-loud fraction with redshift is also consistent with the observed correlation between radio and X-ray emission for faint radio sources (Brinkmann et al. 1995).

These results are surprising in terms of the standard paradigm of AGN energy production. The constancy of the radio luminosity distribution over more than two orders of magnitude in optical luminosity is unexpected in the context of the standard black hole model. The radio emission mechanism appears to be only weakly tied to the mass accretion rate which presumably drives the optical and near-UV emission. Also, the radio-loud fraction is essentially constant over $90 \%$ of the Hubble time, during which the epoch of quasar formation occurs, and the comoving space density declines by a factor of several hundred to the present value.

This work was supported by NSF grant AST 93-20715 and NASA grant NGT-51152, a NASA Graduate Student Fellowship (EJH).

\section{References}

Brinkmann, W., et al. 1995, Ast.Ap.Supp., 109, 147

Dunlop, J. S., \& Peacock, J. A. 1990, M.N.R.A.S., 247, 19

Hooper, E. J., Impey, C. D., Foltz, C. B., \& Hewett, P. C. 1995, Ap.J., 445, 62 (Paper II)

Kellermann, K. I., Sramek, R., Schmidt, M., Shaffer, D. B., \& Green, R. 1989, A.J., 88, 1195 (PG)

Stocke, J. T., et al. 1991, Ap.J.Supp., 76, 813

Visnovsky, K. L., Impey, C. D., Foltz, C. B., Hewett, P. C., Weymann, R. J., \& Morris, S. L. 1992, Ap.J., 391, 560 (Paper I) 